\title{
Body packing: tráfico de drogas y cirugía. Reporte de un caso
}

\author{
Luis Meneses C. ${ }^{1}$ y Javier Meneses P. ${ }^{2}$
}

'Campo Clínico Hospital el

Pino, Universidad Andrés

Bello.

${ }^{2}$ Estudiante de Medicina Campo Clínico Hospital e

Pino, Universidad Andrés Bello. Santiago, Chile.

Recibido 2020-09-28 y aceptado 2020-11-03

Correspondencia a:

Dr. Luis Meneses C. cirujano.rockero@gmail.com

\section{Body packing: drug traffic and surgery. Case report}

\begin{abstract}
Aim: To present the case of a patient with acute cocaine intoxication in the context of body packing syndrome, taking into consideration its medical-surgical and legal aspects. Materials and Method: A clinical record with anonymity protection was obtained, from a patient who was admitted to the emergency room and who was subsequently operated on and managed at Hospital El Pino in San Bernardo, Chile. Results: Patient underwent a gastrotomy, an enterotomy and a colotomy after being stabilized from a cardiorespiratory arrest in the emergency room. He was transferred to the Intensive Care Unit (ICU) where he evolved unfavorably. Discussion: Body packing is an infrequent clinical entity that, in a few cases, may develop severe complications that require immediate medical-surgical treatment, in addition to notification to authorities. Conclusion: Considering that both drug use and traffick remain as an important public health concern and that as its consequences may be devastating, it is important to have a
\end{abstract} knowledge on the subject.

Key words: body packing; intoxication; intestinal obstruction; drug traffick; surgery.

\section{Resumen}

Objetivo: Exponer el caso de un paciente con intoxicación aguda por cocaína en contexto de un body packing syndrome, considerando sus aspectos médico-quirúrgicos y legales. Materiales y Método: Se obtuvo la ficha clínica, con resguardo de anonimato, de un paciente que acude al servicio de urgencia (SU) y que es intervenido y evolucionando posteriormente en el Hospital el Pino de San Bernardo, Chile. Resultados: Paciente fue sometido a una gastrotomía, una enterotomía y una colotomía después de haber sido estabilizado por paro cardiorrespiratorio presentado en SU. Es trasladado a unidad de cuidados intensivos (UCI) donde evoluciona desfavorablemente. Discusión: El body packing syndrome es una entidad clínica infrecuente que, en la minoría de los casos, puede presentar complicaciones severas que requieren de un tratamiento médico-quirúrgico inmediato. Conclusión: Considerando que tanto el consumo como el tráfico de drogas se mantienen como un importante problema de salud pública y que sus consecuencias pueden ser devastadoras, es importante tener conocimiento sobre el tema.

Palabras clave: body packing; intoxicación; obstrucción intestinal; tráfico de drogas; cirugía.

\section{Introducción}

El consumo y el tráfico de drogas se mantienen como un importante, y muy prevalente, problema de salud a nivel mundial. En esta línea, y según la United Nations Office on Drugs and Crime (UNODC) $)^{1}$, durante el año 2016, unos 276 millones de personas en el mundo consumieron drogas, entre las que destacan marihuana, heroína y cocaína. Nuestro continente, y sobre todo Latinoamérica, se encuentra importantemente afectado, siendo Colombia, Bolivia y Perú los mayores productores y distribuidores, especialmente de cocaína ${ }^{1,2}$. Chile es un destino final común de muchas de las rutas de narcotráfico, registrándose, hacia nuestro país, un flujo mayoritario de marihuana y cocaína ${ }^{3}$. En tal sentido, una forma común de tráfico de drogas es el llamado body packing, en el que un individuo, coloquialmente apodado como "mula", ingiere voluntaria o forzadamente paquetes de droga con el objetivo de contrabandear ${ }^{4}$.

El primer caso clínico relacionado con body packing se registró en 1971 y se descubrió en el 
curso de una obstrucción intestinal que presentó el paciente por los paquetes de droga ${ }^{5}$. Así, los pacientes pueden concurrir al SU de manera voluntaria o acompañados de personal policial, con grados variables de compromiso, desde un estado asintomático hasta otro potencialmente severo como consecuencia de la rotura o filtración de los paquetes, con intoxicación aguda secundaria y/o de la generación de una obstrucción intestinal por los mismos, sucesos integrados en el término body packer syndrome ${ }^{6}$, que condicionan las indicaciones quirúrgicas más comunes en estos pacientes ${ }^{7}$.

En relación con los cuadros de intoxicación aguda, en específico secundaria a cocaína o heroína, se puede observar un espectro variado de manifestaciones con repercusiones multiorgánicas, particularmente sobre los sistemas cardiovascular (CV) y nervioso central (SNC), además de respiratorio en el caso de la heroína ${ }^{7-9}$. La intoxicación por cocaína se manifiesta principalmente con agitación, ictus y, en casos graves coma; además de isquemia coronaria, arritmias potencialmente mortales como taquicardia ventricular, paro cardíaco y muerte súbita ${ }^{8}$. Por otro lado, aquella secundaria a heroína cursa con depresión del SNC, manifestando desde somnolencia hasta coma, depresión e insuficiencia respiratoria, edema pulmonar agudo, hipotensión y bradicardia?

A continuación, presentaremos el caso de un paciente llevado a cirugía abierta posterior a un cuadro de intoxicación aguda, probablemente secundario a cocaína, que evoluciona insatisfactoriamente en posoperatorio y cuidados posteriores.

\section{Caso Clínico}

Paciente masculino de 39 años, extranjero, sin documentos de identidad nacionales y sin antecedentes conocidos, ingresa al servicio de urgencias presentando paro cardiorrespiratorio en el TRIAGE. Se ingresa a sala de reanimación lográndose retorno a circulación espontánea tras 9 min de reanimación cardiopulmonar.

Al examen físico destaca mal estado general, pupilas midriáticas arreactivas e inestabilidad hemodinámica, por lo que se comienza reposición de fluidos y apoyo con drogas vasoactivas, además tenía el abdomen distendido y ruidos hidroaéreos ausentes por lo que se realizó una radiografía de tórax y abdomen como parte de la evaluación inicial que sugirió la presencia de elementos de aspecto ovoide en estómago e intestino delgado (Figura 1), los que fueron confirmados con tomografía computarizada de abdomen y pelvis (Figura 2) y gastroscopía.

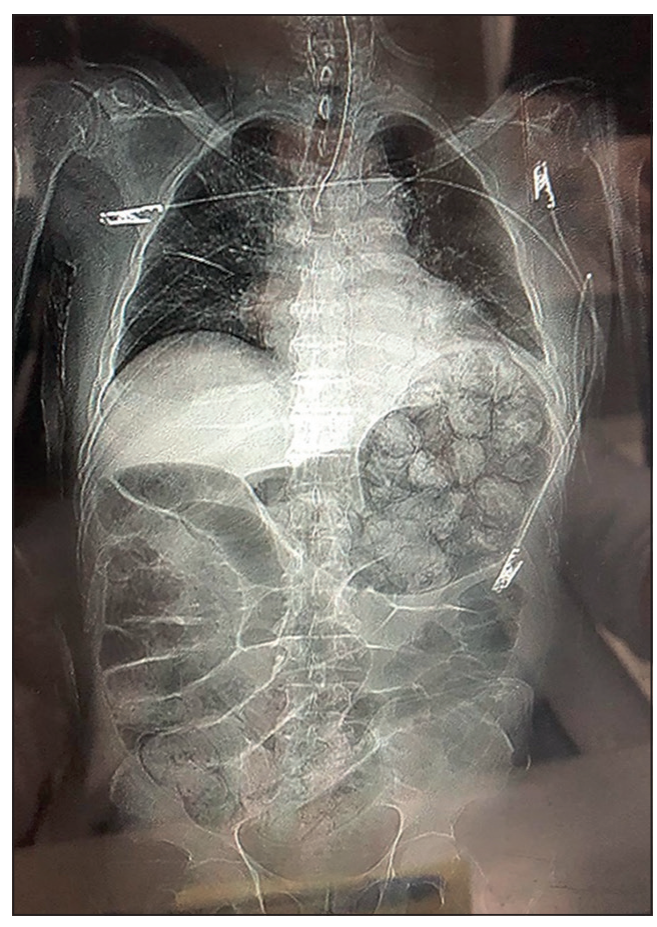

Figura 1. Radiografía de tórax y abdomen que muestra cuerpos extraños de aspecto ovoide en estómago (flecha roja) e intestino delgado (flecha morada)

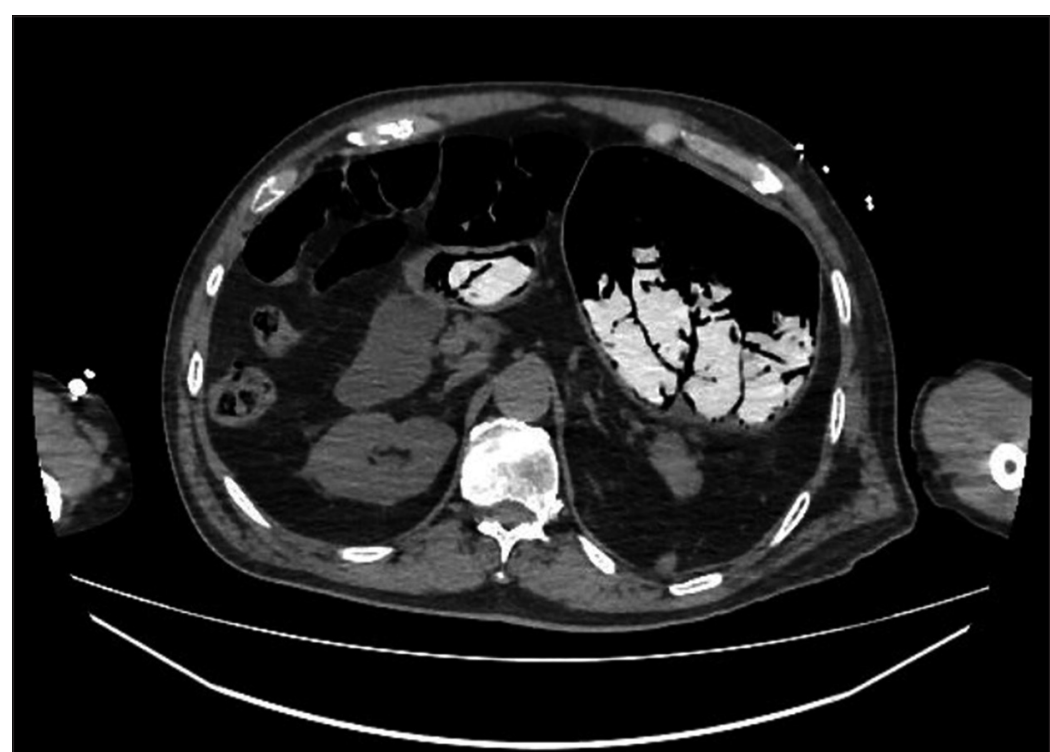

Figura 2. TC de abdomen que evidencia cuerpos extraños hiperdensos a nivel de estómago y primera porción del duodeno.

Una vez estabilizado, por hallazgos y estado crítico del paciente, se decide extraer quirúrgicamente los ovoides. Además, se contacta a autoridades del hospital y a Carabineros de Chile, quienes dan aviso a fiscal de turno sobre el caso. 
En pabellón se realizó laparotomía suprainfraumbilical, encontrándose distensión de estómago y asas delgadas. A continuación, se procedió a realizar una gastrotomía longitudinal a nivel del cuerpo y se extrajeron dieciocho ovoides. La exploración de intestino delgado fue precedida por una maniobra de Kocher y no se palparon ovoides en segmento duodenal. Luego, distal al ángulo de Treitz a nivel de intestino medio, se encontraron diez más que fueron extraídos a través de enterotomía en la unión yeyuno-ileal (Figura 3). Se procedió a revisar colon y recto, encontrándose un ovoide más en el ciego que fue extraído a través de una colotomía en su tenia. Posteriormente se realizó aseo y cierre de la cavidad abdominal. Por último, se llevó a cabo un tacto rectal sin encontrarse ningún otro ovoide. El recuento final fue de 29 unidades y el procedimiento fue presenciado constantemente por tres funcionarios de la policía.

Después de la cirugía el paciente fue trasladado a UCI en malas condiciones generales, destacando acidosis metabólica, coagulopatía y falla orgánica múltiple. Evoluciona con compromiso respiratorio, hepático, renal y cerebral de tipo hipóxico-isquémico severo durante el primer mes. Evolutivamente fue evaluado por equipo multidisciplinario que determinó irreversibilidad del cuadro clínico y además se consultó con comité de ética asistencial de la institución que sugirió limitar tratamiento e indicación de exámenes, ofrecer medidas básicas de soporte clínico y otorgar apoyo social. El paciente falleció días después.

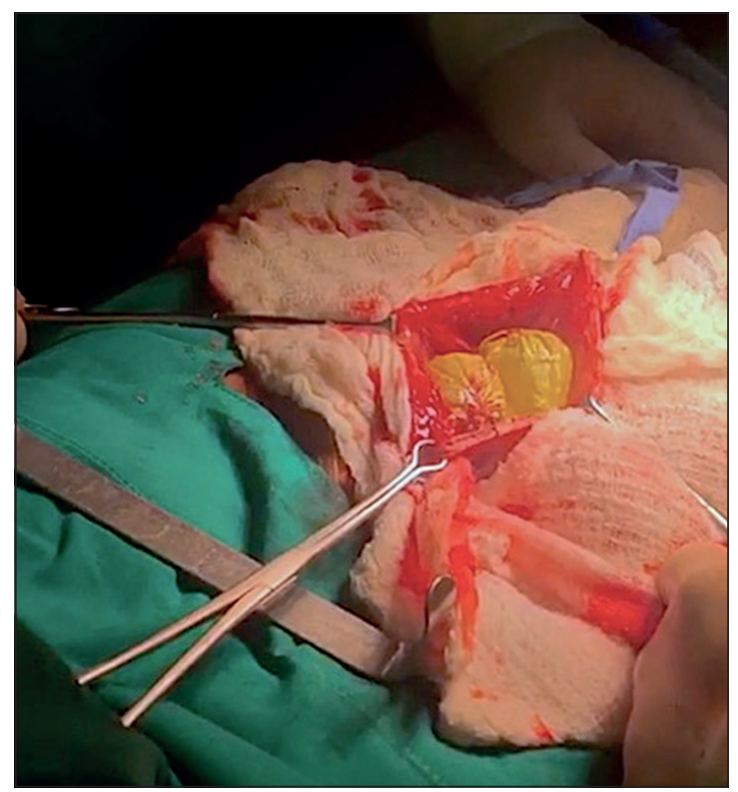

\section{Discusión}

El body packing como método de tráfico de drogas es una práctica cada vez más utilizada a nivel mundial, sin embargo, no existen estudios epidemiológicos de gran escala que lo describan, por lo que contamos solo con series puntuales que nos sugieren su importancia, como una que se realizó en el Aeropuerto Internacional de Nueva York, en la cual, entre los años 1993 y 2005, se detectaron 1.250 casos $^{10}$. Por otra parte, la presentación clínica de estos pacientes se ha mantenido relativamente constante a lo largo del tiempo, comportamiento que puede observarse en tres series de casos, de los años $1983^{11}, 2002^{6}$ y $2009^{12}$, en las que se describe que la presentación más frecuente en algún servicio de salud es asintomática (75\%, 88,2\% y $66,6 \%$, respectivamente), seguida de obstrucción intestinal e intoxicación por fuga o rotura de los paquetes. Esta última presentación, que concuerda con la del caso que presentamos, ha disminuido en frecuencia, probablemente por el hecho de que los métodos de envasado son cada vez más sofisticados pasando desde, antiguamente, condones o bolsas de plástico atadas en un extremo hacia envolturas con varias capas de látex recubiertas por cera, más utilizadas actualmente ${ }^{10}$.

El enfoque inicial frente a todo paciente debe comprender la evaluación de factores que puedan determinar complicaciones severas como lo son: la naturaleza de la droga, cantidad, integridad y localización de los paquetes, tiempo que han permanecido en el tracto gastrointestinal (TGI) y uso de fármacos anticinéticos ${ }^{13}$.

A grandes rasgos, el manejo de estos pacientes puede ser conservador (principalmente en pacientes asintomáticos) o quirúrgico (indicado en todo paciente que presente clínica de obstrucción intestinal o intoxicación). El primero debe incluir monitorización cercana en UCI y considerar uso de irrigación gastrointestinal con polietilenglicol, además de algún fármaco procinético como metoclopramida. El manejo quirúrgico debe ser inmediato en cuadros de intoxicación aguda, aunque es conveniente manejar la hipertermia, hipertensión arterial, convulsiones y arritmias con nitroglicerina, midazolam y amiodarona, preoperatoriamente ${ }^{10,12}$.

En cuanto al método quirúrgico se describen tres opciones principalmente: 1) "exprimir" TGI con fin de vaciar mecánicamente; 2) enterotomía única hacia la que se "exprimen" los paquetes y 3) múltiples enterotomías con extracción directa, dependiendo de cuál sea la realidad a la que se enfrenta el equipo y considerando el riesgo de infección del sitio quirúrgico, que es la principal complicación ${ }^{12}$. 
El caso presentado fue la primera experiencia de este tipo en nuestro equipo médico-quirúrgico y su enfrentamiento involucró un manejo inicial (ABCDE) con estabilización y posterior resolución quirúrgica a través de gastrotomía, enterotomía y colotomía, sin complicaciones derivadas de la técnica quirúrgica. La presentación y posterior evolución concuerda con la que se produce en los casos de intoxicación por cocaína, que involucra, principalmente, compromiso $\mathrm{CV}$ y del SNC que, en una minoría de casos, como ocurrió con nuestro paciente, tiene un desenlace fatal.

Por último, queremos destacar el importante aspecto medicolegal que involucra este caso y que se detalla en la Ley 20.000 artículo 13. En esta se menciona, en síntesis, que todo funcionario público de Chile que tome conocimiento de algún delito que involucre la ley (tráfico de drogas) debe denunciarlo o, de lo contrario, será castigado con presidio y multa $^{14}$.

\section{Conclusión}

Tanto el consumo como el tráfico de drogas prevalecerán como importantes problemas de salud pública a nivel mundial, por lo que es esperable que casos como este se mantengan o aumenten en frecuencia. No siendo frecuente recibir y enfrentar pacientes de este tipo, siempre revisten una complejidad mayor por las potenciales, complejas y eventualmente mortales complicaciones que pueden tener, sobre todos aquellos con intoxicación aguda, por lo que se requiere un conocimiento y manejo adecuado, sin olvidar el importante aspecto medicolegal que involucra.

\section{Responsabilidades éticas}

Protección de personas y animales. Los autores declaran que para esta investigación no se han realizado experimentos en seres humanos ni en animales.

Confidencialidad de los datos. Los autores declaran que en este artículo no aparecen datos de pacientes.

Conflictos de interés: no hay.

\section{Bibliografía}

1. UNODC. Informe mundial sobre las drogas 2018 [Internet]. 2018. Available from: https://www.unodc.org/wdr2018/ prelaunch/WDR18_ExSum_Spanish.pdf [citado el 19 de septiembre de 2020].

2. Greene P, Fynmore S, Vinagre A. Serie informe sociedad y política. Drogas en Chile: Fronteras, consumo e institucionalidad [Internet]. 2018. Available from: https://lyd.org/wpcontent/uploads/2018/03/SIP-161Drogas-en-Chile-Fronteras-consumo-einstitucionalidad-Enero2018.pdf [citado el 21 de septiembre de 2020].

3. Fiscalía MP de C. Observatorio del narcotráfico, informe 2020 [Internet]. 2020. Available from: http://www. fiscaliadechile.cl/Fiscalia/quienes/ observatorio_narcotrafico_informe_2020. pdf [citado el 21 de septiembre de 2020].

4. O'Malley GOR. Transporte de drogas ilegales en el interior del cuerpo (body stuffing y body packing) - Temas especiales - Manual MSD versión para profesionales [Internet]. 2018. Available from: https://www.msdmanuals.com/es-cl/ professional/temas-especiales/drogasrecreativas-y-tóxicas/transporte-dedrogas-ilegales-en-el-interior-del-cuerpobody-stuffing-y-body-packing [citado el 21 de septiembre de 2020].

5. Deitel MSA. Intestinal obstruction by an unusual foreign body [Internet]. 1973 Available from: https://pubmed.ncbi. nlm.nih.gov/4728949/ [citado el 21 de septiembre de 2020].

6. Pidoto RR, Agliata AM, Bertolini R, Mainini A, Rossi G, Giani G. A new method of packaging cocaine for international traffic and implications for the management of cocaine body packers. J Emerg Med. [Internet]. 2002;23:149-53. Available from: https:// linkinghub.elsevier.com/retrieve/pii/ S073646790200505X [citado el 21 de septiembre de 2020].

7. Elkbuli A, Ehrhardt JD, Hai S, McKenney M, Boneva D. Surgical care for ingested cocaine packets: Case report and literature review. Int J Surg Case Rep. [Internet]. 2019;55:84-7. Available from: https:// linkinghub.elsevier.com/retrieve/pii/ S2210261219300240 [citado el 21 de septiembre de 2020].
8. Nogué Xarau S, Picón Cabrera M, G. Mestre Roca R. Corcuera Romero de la Devesa. Urgencias en usuarios de cocaína [Internet]. 2002. Available from: https:// www.elsevier.es/es-revista-medicinaintegral-63-pdf-13029968 [citado el 19 de septiembre de 2020].

9. Porras-Morales G. Intoxicación aguda por heroína [Internet]. 2004. Available from: https://www.scielo. sa.cr/scielo.php?script=sci_arttext\&pid $=$ S0001-60022004000100008 [citado el 21 de septiembre de 2020].

10. Hoffman RS. Internal concealment of drugs of abuse (body packing) UpToDate [Internet]. 2020. Available from: https://www-uptodate-com. recursosbiblioteca.unab.cl/contents/ internal-concealment-of-drugs-of-abusebody-packing?search=Cocaine: Acute into xication\&topicRef $=303 \&$ source $=$ see_link [citado el 21 de septiembre de 2020].

11. McCarron MM, Wood JD. The cocaine "body packer" syndrome. Diagnosis and treatment [Internet]. 1983. Available from: https://pubmed.ncbi.nlm.nih. gov/6887463/ [citado el 21 de septiembre de 2020]. 


\section{CASOS CLÍNICOS}

12. Yegane RA, Bashashati $M$, Hajinasrollah E, Heidari K, Salehi NA, Ahmadi M. Surgical approach to body packing [Internet]. Vol. 52, Diseases of the Colon and Rectum. 2009;97-103. Available from: http://journals.lww.com/00003453-
200901000-00016 [citado el 21 de septiembre de 2020].

13. Madrazo Z. Secanella SL, Golda T. Síndrome de Body Packer por intoxicación por cocaína [Internet]. 2007. Available from: http://scielo.isciii. es/scielo.php?script=sci_arttext\&pid
$=\mathrm{S} 1130-01082007001000017$ [citado el 21 de septiembre de 2020].

14. Ley-20000 16-FEB-2005 Ministerio del Interior [Internet]. Available from: https://www.bcn.cl/leychile/ navegar?idNorma $=235507$ [citado el 21 de septiembre de 2020]. 